\title{
Smallest critical region for microcephaly in a patient with mosaic ring chromosome 13
}

\author{
P.-H. Su ${ }^{1,2}$, C.-P. Chen ${ }^{3}$, Y.-N. Su ${ }^{4}$, S.-J. Chen ${ }^{1}$, L.-L. Lin ${ }^{1}$ and J.-Y. Chen ${ }^{1,2}$ \\ ${ }^{1}$ Department of Pediatrics, Chung Shan Medical University Hospital, \\ Taichung, Taiwan \\ ${ }^{2}$ School of Medicine, Chung Shan Medical University, Taichung, Taiwan \\ ${ }^{3}$ Department of Obstetrics and Gynecology, School of Medicine, \\ National Yang-Ming University, Taipei, Taiwan \\ ${ }^{4}$ Department of Medical Genetics, National Taiwan University Hospital, \\ Taipei, Taiwan \\ Corresponding author: J.-Y. Chen \\ E-mail: jen@csh.org.tw
}

Genet. Mol. Res. 12 (2): 1311-1317 (2013)

Received September 6, 2012

Accepted January 20, 2013

Published April 25, 2013

DOI http://dx.doi.org/10.4238/2013.April.25.2

\begin{abstract}
A ring chromosome 13 or r(13) exhibits breakage and reunion at breakage points on the long and short arms of chromosome 13, with deletions of the chromosomal segments distal to the breakage points. The $\mathrm{r}(13)$ chromosome accounts for approximately $20 \%$ of ring chromosomes compatible with life. We describe a female patient with mental retardation, growth retardation, microcephaly, craniofacial dysmorphy, hearing impairment, and prolonged prothrombin time. Chromosomal analysis via GTG banding of peripheral blood lymphocy tes revealed a karyotype of 46,XX,r(13)(p13q34)[71]/45,XX,-13[12]/ 46,XX,dic r(13;13)(p13q34;p13q34)[9]/46,XX,-13,+mar[5]/47, XX, +r(13) $(\mathrm{p} 13 \mathrm{q} 34) \times 2[2] / 46, X X[1]$ at the age of 6 years and 46,XX,r(13)(p13q34) [82]/45,XX,-13[14]/46,XX, dic r(13;13)(p13q34; p13q34)[2]/46,XX, $-13,+\operatorname{mar}[2]$. Array comparative genomic hybridization analysis of the blood demonstrated a 4.37-Mb deletion on chromosome 13q [arr
\end{abstract}


cgh 13q34q34(109,743,729-144,110,721)]. A cytogenetic study of peripheral blood revealed a rare chromosomal abnormality associated with different cell lines that included structural and numerical abnormalities of chromosome 13 . This case, along with 14 previously reported cases, indicate that the smallest critical region for chromosome 13 microcephaly is $109,743,729-144,110,721$.

Key words: Array comparative genomic hybridization; Microcephaly; Mosaic ring chromosome; Ring chromosome 13 duplication/deletion; Hearing impairment; Prolonged prothrombin time

\section{INTRODUCTION}

Microcephaly is relatively common among developmentally delayed children. Four single etiologic genes have been identified. Microcephaly is also associated with at least 7 loci (Kinsman and Johnston, 2011) and is commonly observed in ring chromosome 13, or r(13) (Brandt et al., 1992; Bedoyan et al., 2004). Ring chromosomes arise through fusion of the telomeric ends or both arms of a chromosome at their breakage points with loss of distal ends. $\mathrm{r}(13)$ occurs in approximately 1/58,000 live births and is more prevalent in females (60:40). The clinical picture of many $\mathrm{r}(13)$ cases is influenced by the stability of the ring and can be similar to or more severe than that in terminal deletions of segments of 13q. The 13q deletion syndrome is caused by deletions including 13q13, 13q14, 13q22, 13q32, 13q33, and 13q34 (Brandt et al., 1992; Mathijssen et al., 2005; Kirchhoff et al., 2009). The current classification is based on the position of chromosomal breakage and fusion. Intellectual disability (mental retardation) and growth deficiency are common in both group 1 (deletions are proximal to 13q32) and group 2 (deletions include q32) (Liao et al., 2011). Common symptoms of group 3 (breakage points in 13q33 and 13q34) include marked intellectual disability, growth delay, and dysmorphic facial features (Liao et al., 2011). Other malformations may involve the limbs, brain, eye, kidney, or heart. In addition, an r(13q34) has been mapped to the $F 7$ gene encoding hematological factor VII (FVII) and the F10 gene encoding FX on distal chromosome $13 q 34$ (Kasai et al., 1989). The levels of FVII and FX in r(13) patients are usually 50\% of normal diploid concentrations (Kasai et al., 1989) and have minimal effects on clotting times (Walczak-Sztulpa et al., 2008).

Chromosome mosaicism is the result of a postzygotic event (Golabi et al., 2010) and involves several distinct cell lines. Herein, we describe a girl with psychomotor disability, distinct facial features, prolonged prothrombin time, and hearing loss. A cytogenetic study of

peripheral blood revealed a rare chromosomal abnormality associated with different cell lines that included structural and numeric abnormalities of chromosome 13.

\section{CASE REPORT}

The proposita was born full term to a 25 -year-old (gravida 3) Taiwanese woman with no known history of diabetes, hypertension, illness, infection, or teratogen. The birth weight $(2060 \mathrm{~g})$ and length $(44 \mathrm{~cm})$ were below the third percentile. The female baby was small for her gestational age and had respiratory distress but no additional perinatal complications. 
Monitoring of developmental milestones through occipitofrontal head circumference began at 18 months at a neurologic clinic. The child was able to sit at 1.3 years and walk without support at 2 years. Her significant delay in speech development prompted an auditory examination that revealed conductive hearing loss of $50 \mathrm{~dB}$. She had amblyopia and hypermetropia.

The girl was referred to our pediatric genetic clinic for evaluation because of psychomotor delay and short stature at the age of 6 years. She had intellectual disability and craniofacial dysmorphisms including microcephaly, a narrow and sloping forehead, downslanting eyebrows, epicanthal folds, hypertelorism, malformed ears, small mandible, short philtrum, and opening mouth (Figure 1). Additionally, she had small hands and club feet. Her height was $107 \mathrm{~cm}$ (third percentile), and her weight was $19 \mathrm{~kg}$, which was between the 25th and 50th percentile. Bone age determined from a radiograph of the left hand and wrist according to the standards of Greulich and Pyle (1959) was 4 years. A whole-spine survey revealed scoliosis along the thoracic to lumbar spine. A magnetic resonance imaging scan of the brain revealed bilateral leukomalacia. Sonograms of the heart, kidney, and liver revealed normal structures. Her verbal IQ (65), performance IQ (58), and functional IQ (58) were low.

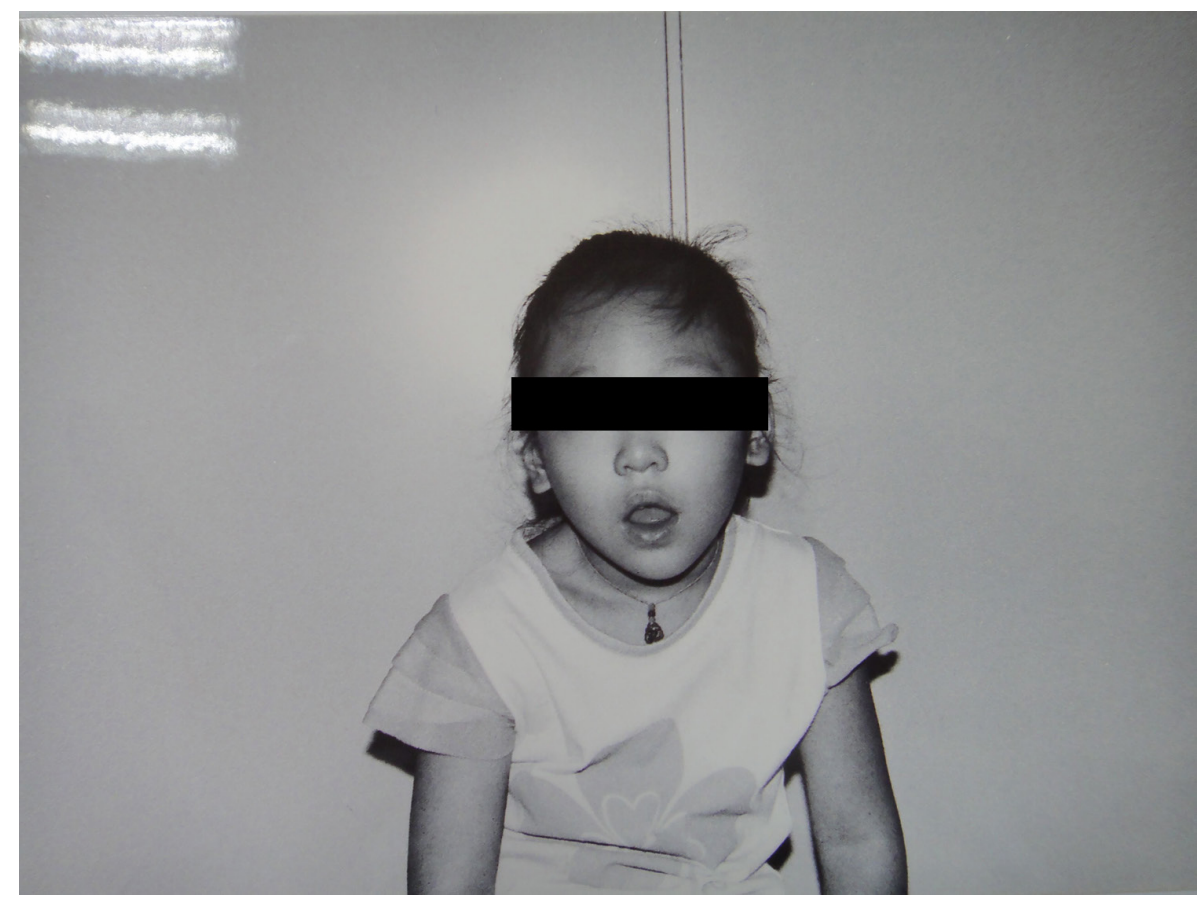

Figure 1. Photo of the patient showing microcephaly, a narrow and sloping forehead, downslanting eyebrow, epicanthic folds, hypertelorism, malformed ears, small mandible, short philtrum, and open mouth.

Frequent nasal bleeding, snoring, and sleeping problems at age 13 prompted a referral to an ear, nose, and throat clinic for tonsilectomy. The patient had a prolonged prothrombin time of $13.7 \mathrm{~s}$ compared with the normal range of 8.0-12.0 s. The activated partial thromboplastin time of $34.9 \mathrm{~s}$ and bleeding time of $1 \mathrm{~min}$ were normal. Her menarche began at 14.5 years and was normal. Written consent from parents was obtained. 
GTG banding was performed on 100 metaphases from peripheral blood at 6 and 14 years of age. Array comparative genomic hybridization $(\mathrm{aCGH})$ was performed on peripheral blood mononuclear cells using Cytochip ${ }^{\mathrm{TM}}$ Oligo (Blue Gnome, Cambridge, UK) according to manufacturer instructions, and the chip was scanned with a high-resolution scanner at $5 \mu \mathrm{m}$ for quantitative analysis. Quality assessment was based on the median absolute pairwise difference of each chip.

Chromosomal analysis through GTG banding of the blood at age 6 years showed 46,XX,r(13)(p13q34)[71]/45,XX,-13[12]/46,XX,dic r(13;13)(p13q34;p13q34)[9]/46,XX,-13, $+\operatorname{mar}[5] / 47, \mathrm{XX},+\mathrm{r}(13)(\mathrm{p} 13 \mathrm{q} 34) \times 2[2] / 46, \mathrm{XX}[1]$. A second chromosomal analysis via GTG banding of peripheral blood mononuclear cells at 14 years was comparable: 46,XX,r(13) (p13q34)[82]/45,XX,-13[14]/46,XX,dic r(13;13)(p13q34;p13q34)[2]/46,XX,-13,+mar[2] (Figure 2). Chromosome 13 in the peripheral blood mononuclear cells showed mosaicism, which included r(13) (82\%), monosomy chromosome 13 (14\%), dicentric chromosome 13(2\%), and chromosome 13 mar (2\%). Oligonucleotide-based aCGH analysis of the blood using Cytochip $^{\mathrm{TM}}$ Oligo (Blue Gnome) demonstrated a 4.37-Mb deletion at chromosome 13q (arr cgh 13q34q34[109,743,729-144,110,721] $\times 1$ ) (Figure 3). This deletion was located in the 13q34-qter and deleted the following genes: COL4A1, COL4A2, RAB20, CARKD, CARS2, ING1, ANKRD10, ARHGEF7, C13orf76, SOX1, C13orf28, TUBGCP3, ATPI1A, MCF2L, F7, F10, PROZ, PCID2, CUL4A, LAMP1, GRTP1, ADPRHL1, DCUNID2, TMCO3, TFDP1, $A T P 4 B$, GRK1, GAS6, FAM70B, RASA3, CDC16, UPF3A, and ZNF828. The karyotypes of both parents were normal.

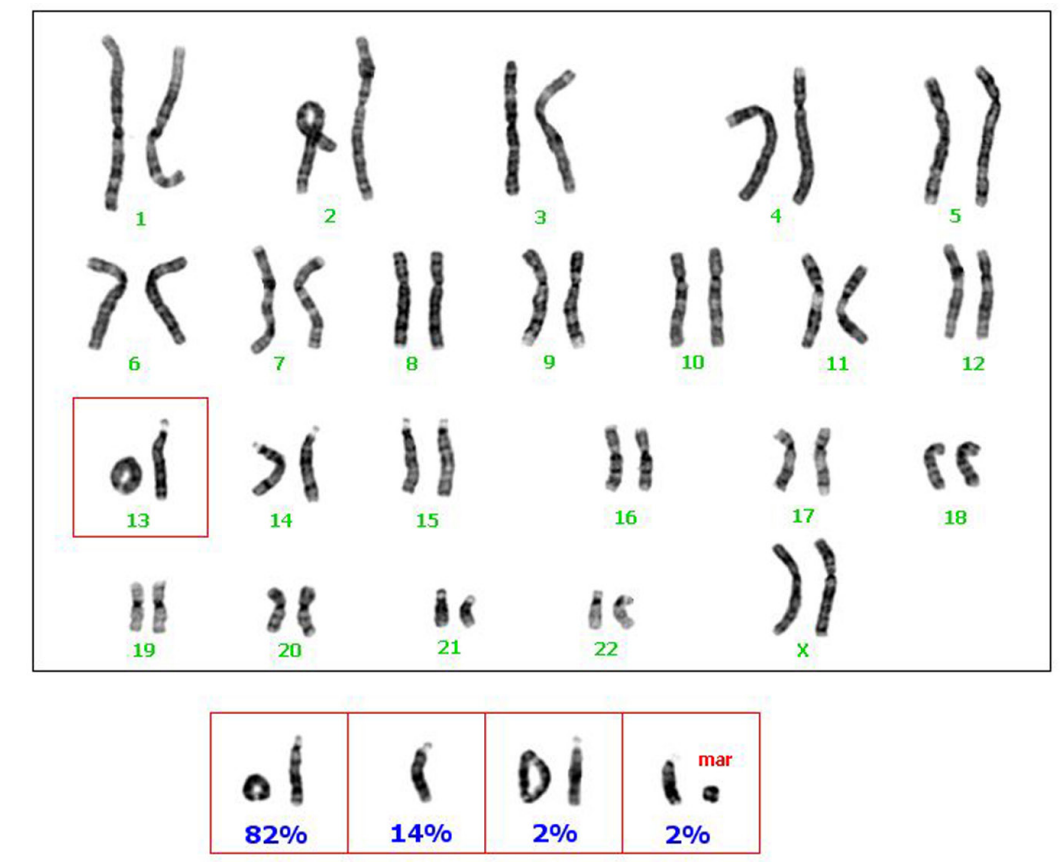

Figure 2. Karyotype of the 6-year-old patient by GTG banding of the peripheral blood showed mosaicism on chromosome 13. The ring chr13 (82\%), chr13q34 deletion, chr 13 trisomy (2\%), and chr 13 mar (2\%) comprised the mosaicism. 


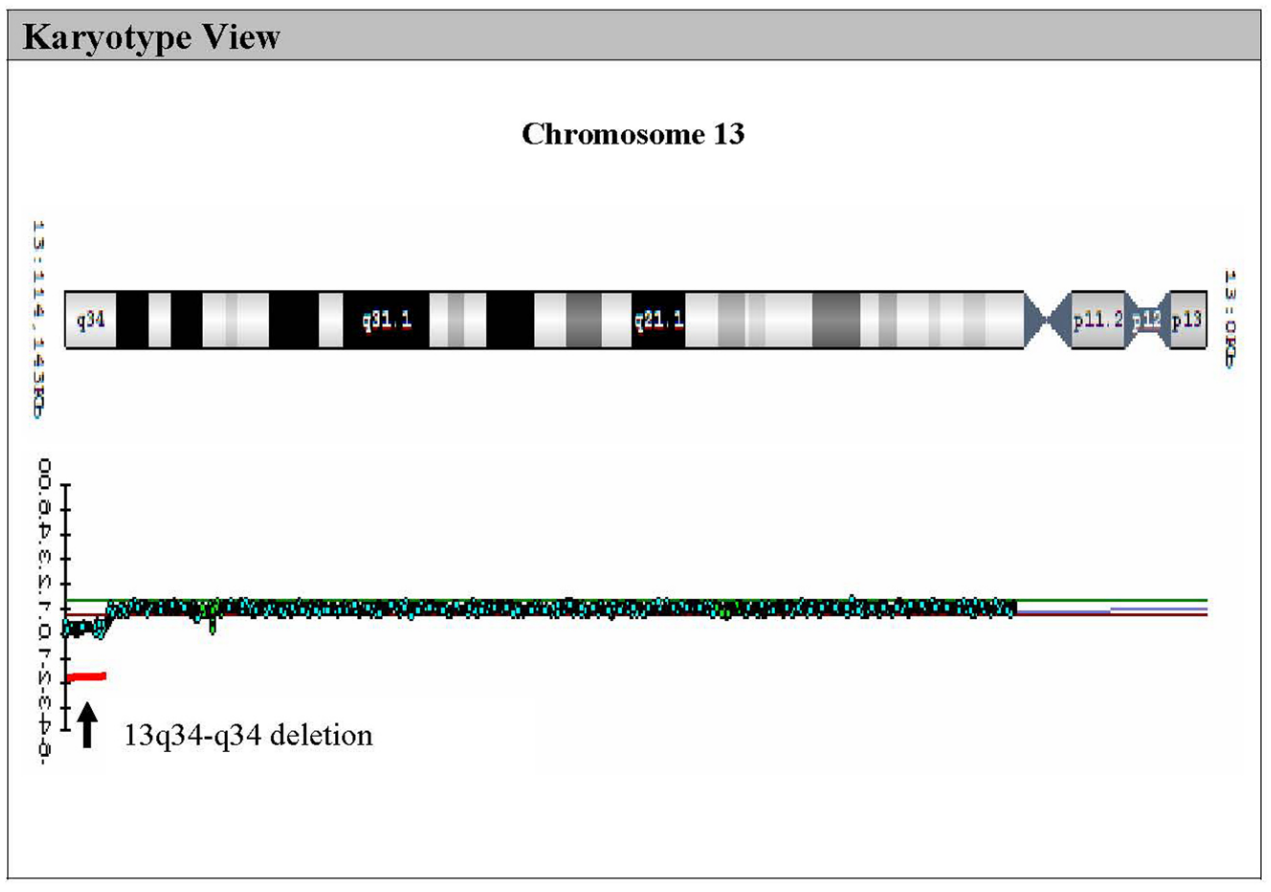

Figure 3. aCGH revealed a 4.37-Mb deletion at chr13q34.

\section{DISCUSSION}

Features of r(13) include intellectual disability, facial dysmorphisms, microcephaly, and hypertelorism. Clinical features of $\mathrm{r}(\mathrm{chr} 13)$ cases are summarized in Table 1 . We have demonstrated the usefulness of aCGH for prenatal detection of microdeletions and unbalanced translocation (Chen et al., 2009a,b). We have also demonstrated the utility of aCGH in the determination of breakage points in a ring chromosome 18, which comprises the majority of mosaicism (Chen et al., 2010). In an analogous manner, aCGH detected a 4.37-Mb 13q microdeletion in this $\mathrm{r}(13)$ case. However, aCGH was unable to detect the low-level mosaicism for disomy 13, r(13) duplication, and $\mathrm{r}(13)$ deletion in this case.

Although $F 7$ and $F 10$ were deleted, the low prothrombin time in this case was unexpected because most patients with $\mathrm{r}(13) \mathrm{q}$ produce sufficient levels of FVII and FX with the unaffected chromosome 13 (Walczak-Sztulpa et al., 2008). In agreement with our case, Kurosawa et al. (2009) have described a female infant with a 13 q 32 deletion and $25 \%$ levels of FVII and FX. It is feasible that additional mutations in the intact chromosome 13 may affect FVII and FX production or clotting efficiency. The patient described herein also displayed hearing loss, which has been observed in another $\mathrm{r}(13)$ patient (Liao et al., 2011), in a patient with chromosome 13 disomy (Yan et al., 2007), and in 2 of 13 patients with chromosome 13 deletions (Kirchhoff et al., 2009). Adding our case to those described by Kirchhoff et al. (2009), the minimal critical deletion for microcephaly is $13 \mathrm{q} 34$. 


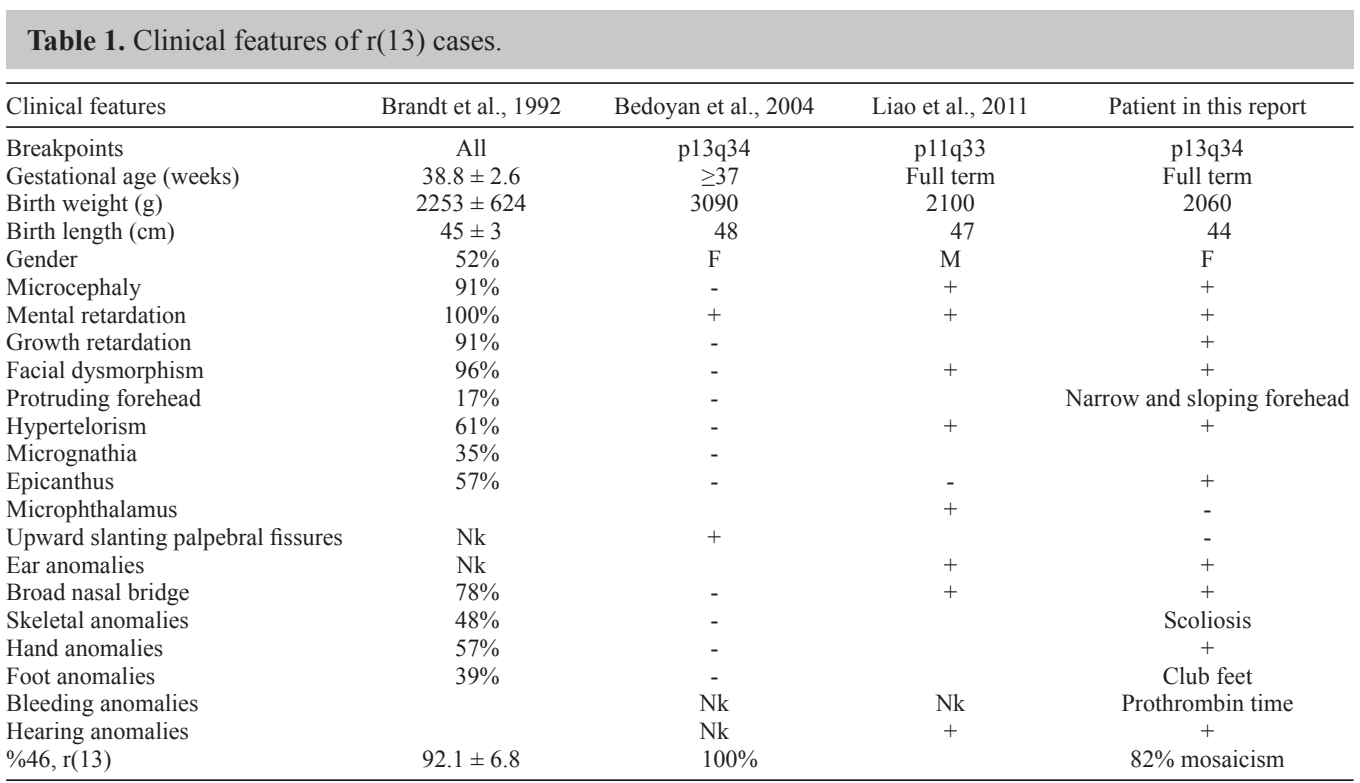

$\mathrm{F}=$ female; $\mathrm{M}=$ male; $\mathrm{Nk}=$ not known.

In summary, patients with $13 \mathrm{q} 34$ deletions have craniofacial abnormalities and psychomotor retardation as well as other specific anomalies, including those of the eyes, ear, limbs, and coagulation factors. We suggest careful evaluation of multiple organs for early management. In addition, patients with normal reproductive ability should be counseled about the possibility of transmission to the next generation. Our case, along with the 14 cases described by Kirchhoff et al. (2009), narrows the critical region for microcephaly to $13 \mathrm{q} 34$.

\section{REFERENCES}

Bedoyan JK, Flore LA, Alkatib A, Ebrahim SA, et al. (2004). Transmission of ring chromosome 13 from a mother to daughter with both having a 46,XX, r(13)(p13q34) karyotype. Am. J. Med. Genet. A 129A: 316-320.

Brandt CA, Hertz JM, Petersen MB, Vogel F, et al. (1992). Ring chromosome 13: lack of distinct syndromes based on different breakpoints on 13q. J. Med. Genet. 29: 704-708.

Chen CP, Su YN, Chang TY, Chern SR, et al. (2009a). 22q11.2 microdeletion in a fetus with double-outlet right ventricle, pulmonary stenosis and a ventricular septal defect: prenatal diagnosis by array comparative genomic hybridization. Taiwan. J. Obstet. Gynecol. 48: 437-440.

Chen CP, Su YN, Tsai FJ, Lin HH, et al. (2009b). Terminal 2q deletion and distal 15q duplication: prenatal diagnosis by array comparative genomic hybridization using uncultured amniocytes. Taiwan. J. Obstet. Gynecol. 48: 441-445.

Chen CP, Kuo YT, Lin SP, Su YN, et al. (2010). Mosaic ring chromosome 18, ring chromosome 18 duplication/deletion and disomy 18: perinatal findings and molecular cytogenetic characterization by fluorescence in situ hybridization and array comparative genomic hybridization. Taiwan. J. Obstet. Gynecol. 49: 327-332.

Golabi M, James AW, Good WV and Cotter PD (2010). Tissue-limited mosaicism for monosomy 13. Am. J. Med. Genet. A 152A: 2634-2639.

Greulich W and Pyle S (1959). Radiographic Atlas of Skeletal Development of Hand and Wrist. Stanford University Press, Stanford.

Kasai R, Narahara K, Namba H, Tsuji K, et al. (1989). Mapping of genes encoding coagulation factors VII and X to the distal portion of the $13 \mathrm{q} 34$ by gene dose study in a patient with r(13). Jinrui Idengaku Zasshi 34: 247-250. 
Kinsman SL and Johnston MV (2011). Microcephaly. Nelson Textbook of Pediatrics (Kliegman RM, Stanton BF, St. Geme JW, Schor NF, et al., eds.). Saunders Elsevier, Philadelphia.

Kirchhoff M, Bisgaard AM, Stoeva R, Dimitrov B, et al. (2009). Phenotype and 244k array-CGH characterization of chromosome 13q deletions: an update of the phenotypic map of 13q21.1-qter. Am. J. Med. Genet. A 149A: 894-905.

Kurosawa H, Suzumura H, Okuya M, Fukushima K, et al. (2009). Haemostatic management of surgery for imperforate anus in a patient with 13q deletion syndrome with combined deficiency of factors VII and X. Haemophilia 15: 398-400.

Liao C, Fu F and Zhang L (2011). Ring chromosome 13 syndrome characterized by high resolution array based comparative genomic hybridization in patient with 47, XYY syndrome: a case report. J. Med. Case. Rep. 5: 99.

Mathijssen IB, Hoovers JM, Mul AN, Man HY, et al. (2005). Array comparative genomic hybridization analysis of a familial duplication of chromosome 13q: a recognizable syndrome. Am. J. Med. Genet. A 136: 76-80.

Walczak-Sztulpa J, Wisniewska M, Latos-Bielenska A, Linne M, et al. (2008). Chromosome deletions in 13q33-34: report of four patients and review of the literature. Am. J. Med. Genet. A 146: 337-342.

Yan D, Ouyang XM, Angeli SI, Du LL, et al. (2007). Paternal uniparental disomy of chromosome 13 causing homozygous 35delG mutation of the GJB2 gene and hearing loss. Am. J. Med. Genet. A 143: 385-386. 